\title{
School absence data for influenza surveillance: a pilot study in the United Kingdom
}

W P Schmidt (Wolf-Peter.Schmidt@lshtm.ac.uk) ${ }^{1}$, R Pebody², P Mangtani ${ }^{1}$

1. Department of Infectious and Tropical Diseases, London School of Hygiene and Tropical Medicine, London, United Kingdom

2. Health Protection Agency, London, United Kingdom

Citation style for this article: Schmidt WP, Pebody R, Mangtani P. School absence data for influenza surveillance: a pilot study in the United Kingdom. Euro Surveill. 2010;15(3):pii=19467. Available online: http://www.eurosurveillance.org/ViewArticle.aspx?Articleld=19467

This article has been published on 21 January 2010

School-age children are at a high risk of acute respiratory virus infections including the 2009 pandemic influenza $A\left(\mathrm{H}_{1} \mathrm{~N}_{1}\right)$. School absence records have been suggested as a tool for influenza surveillance. We analysed absence records from six primary schools (children aged from around five to 11 years) in London during the years 2005 to 2007 in order to provide baseline epidemiological characteristics of illness-related school absence, and to correlate school absence with seasonal influenza. The daily average prevalence of absence due to illness was $2.9 \%$. The incidence was $1.3 \%$ per person-day. The mean duration of absence was 1.8 days (SD 1.8). Over $60 \%$ of absence episodes lasted for one day. Absence prevalence did not differ by sex. Prevalence was highest in the youngest children and then declined slightly, but was again high again in the oldest. Absence was slightly higher on Mondays and Fridays. In general, peaks of absenteeism coincided with peaks of influenza A and B (laboratory reports) but several high peaks were not associated with influenza. There was a better correlation between absence and laboratory reports and prevalence compared to incidence. School absence data may be useful for the detection of localised school outbreaks and as an additional surveillance tool but are limited by lack of data on weekends and during holidays.

\section{Introduction}

School-age children are at high risk of acute respiratory virus infections, in particular influenza, and bear a substantial burden of influenza-related morbidity [1-3]. The 2009 pandemic influenza $A\left(\mathrm{H}_{1} \mathrm{~N}_{1}\right)$ virus has been shown to affect children stronger than adults in terms of attack rate and disease severity $[4,5]$. Furthermore, schools have been recognised as playing a major role in the spread of influenza during an epidemic, and are therefore of particular public health importance for the control of influenza [6-8]. Respiratory infections in general are the leading cause for school absence $[9,10]$, records of which have been suggested as a suitable surveillance tool for influenza [11]. Epidemics of influenza A and B have led to large outbreaks with reported attack rates in one study ranging between $14 \%$ and $42 \%$ [11].
In the United Kingdom (UK), school absence data from a network of boarding schools (Medical Officers of Schools Association - MOSA) have long been used for influenza surveillance. UK state schools are legally required to keep a timely electronic record of school absence. The Health Protection Agency (HPA) has recognised this data source as a promising tool for influenza surveillance [12]. There is evidence that even large school outbreaks of respiratory and influenzalike illnesses can go undetected by other surveillance tools [11]. An HPA pilot study using UK school absence records for influenza surveillance from 11 schools, found that school absence peaked earlier than influenza activity estimated from other surveillance data sources, and is potentially a suitable early indicator of rising incidence [12].

The aim of this analysis was to provide a descriptive analysis of some of the epidemiological characteristics of school absence to inform about the general suitability of routine school absence data in the UK for influenza surveillance. A further aim was to explore whether incidence of school absenteeism is a better measure for surveillance than prevalence which is easier to collect.

\section{Methods \\ Study population}

We collected data from six primary schools from a large borough in east London. We contacted schools from a list of all 52 primary state schools in the area attended by children aged 5 to 12 years. Schools were purposively sampled to cover a wide range of ethnic and socio-economic backgrounds. Receiving free school meals was used as an indicator of socio-economic background of pupils of the respective institutions. A high proportion of pupils of individual schools receiving free school meals was considered as low socio-economic background. Around $60 \%$ of the schools contacted declined inclusion in the study. The final sample included six primary schools, of which four covered school years 1 to 6 , while two schools were infant schools only (years 1 and 2). 
Electronic absence records in the study area contain information on the date and duration of the absence episode as well as the general reason for absence. Detailed information on reasons for illness is not routinely collected or captured electronically. Illness and medical appointments are coded as separate categories. The category "medical appointment" largely refers to planned appointments, for example due to chronic illness. Only "illness" data were included in the analysis.

The electronic absence records of the schools included in the study were converted into Excel spreadsheets FIGURE 1

Duration of absence in six selected schools, United

Kingdom, school years 2005-6 and 2006-7

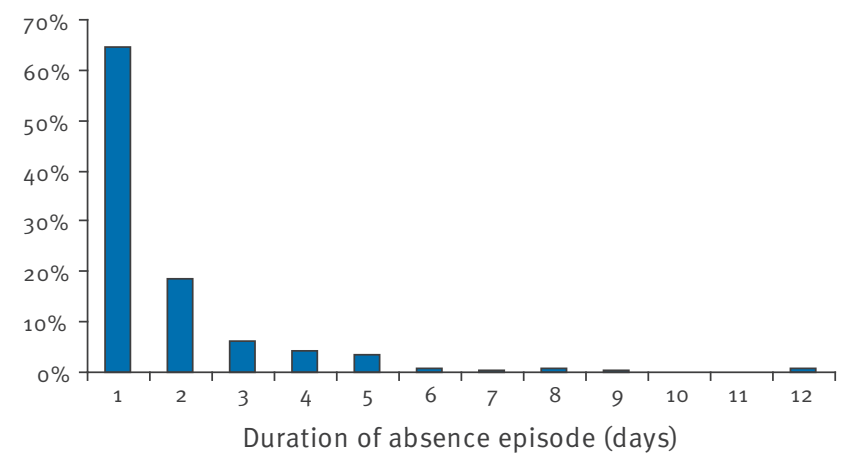

\section{FIGURE 2}

Absence prevalence in six selected schools by school year (class attended), United Kingdom, school years 2005-6 and 2006-7

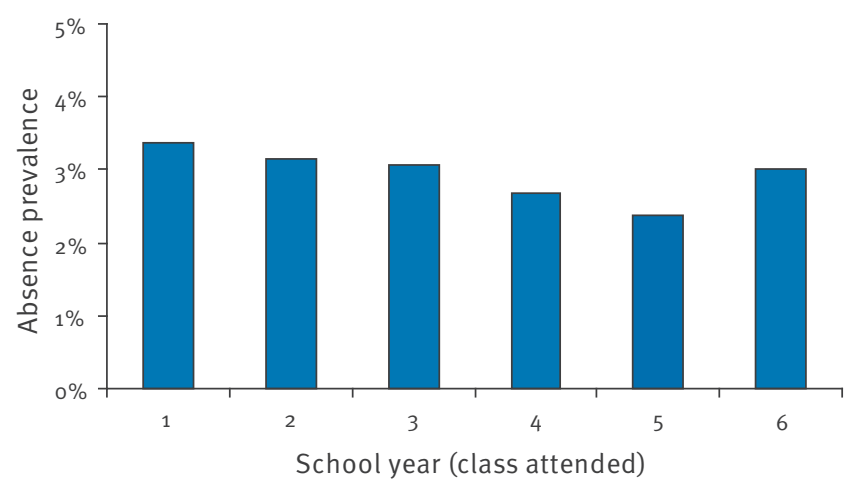

\section{FIGURE 3}

Absence prevalence in six selected schools by week day,

United Kingdom, school years 2005-6 and 2006-7

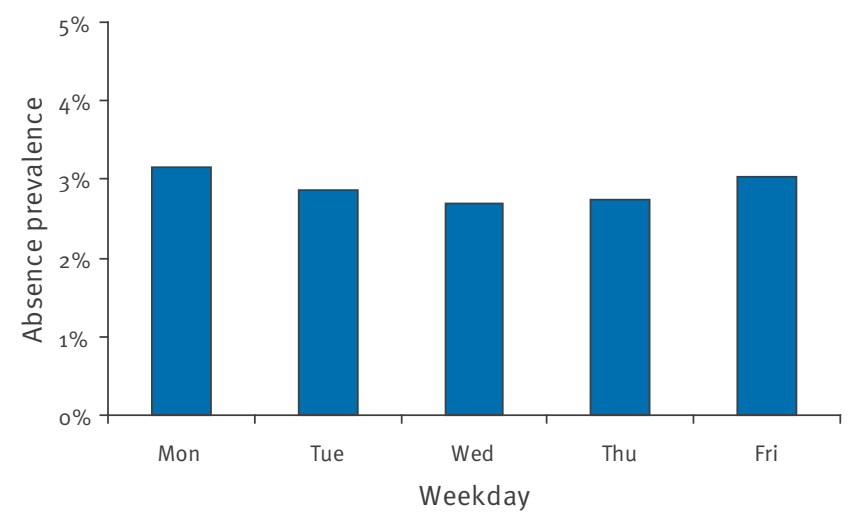

at class level and anonymised before saving them on a removable disk. We retained information on date of absence, school year, and sex and collated the data to obtain all absence records over time at the level of the individual child. From these data we calculated the daily prevalence of absence as well as the incidence of absence. Incidence was defined as new cases of absence among children at risk of being absent on a given day. For the calculation of incidence, children who were already ill the previous day were excluded from the denominator. Children reported ill on Friday and the following Monday were treated as having experienced one episode of absence. Children reported ill on the last day before and the first day after school holidays of one week or longer were treated as having experienced two separate episodes.

Influenza surveillance data for the seasons 2005-6 and 2006-7 were provided by the HPA in Colindale, London. For the analysis we included laboratory reports of confirmed influenza $A$ and $B$ cases in the regions London, Southwest England, Southeast England and East England.

The analysis was restricted to the school years 2005-6 and 2006-7 because most schools were unable to provide data prior to these years. We conducted descriptive statistics, and simple graphical descriptive analysis of the relationship between school absence and influenza laboratory reports. The correlation between daily influenza laboratory reports ( $A$ and $B$ combined) and school absence at different lag times was explored using cross-correlation analysis. The analysis was done in STATA 10.

\section{Results}

Among the six participating schools, the mean number of children per school was 391 (range 187 to 660). The children in these schools were from various ethnic backgrounds (mainly South Asian, West African, Arabic, Eastern European, White British). The proportion of pupils receiving free school meals ranged from $8 \%$ and $30 \%$.

\section{FIGURE 4}

Absence prevalence in six selected schools by proportion of children receiving free school meals, United Kingdom, school years 2005-6 and 2006-7

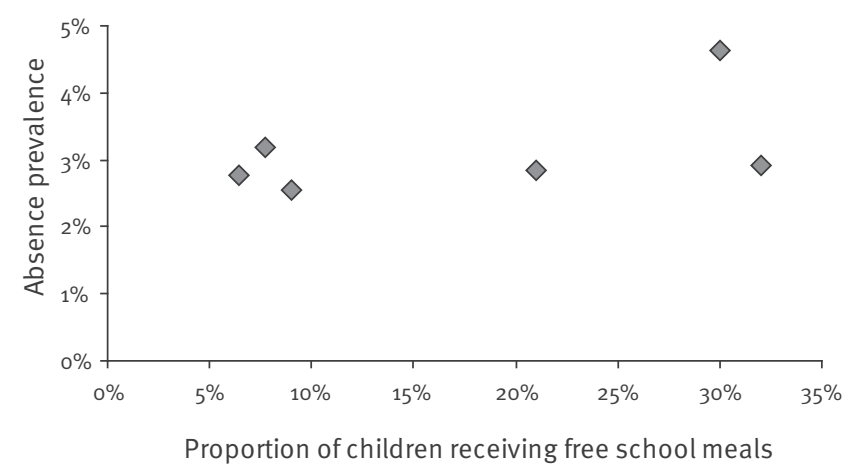


Overall, the prevalence of school absence due to illness per day was $2.9 \%$ (inter-school range $2.5 \%$ to $4.6 \%$ ). The incidence of school absence was $1.3 \%$ per person-day. The mean duration of absence was 1.8 days (standard deviation 1.8). Most absence episodes ( $>60 \%$ ) lasted for only one day (Figure 1). Absence prevalence did not differ by gender (girls $2.8 \%$, boys $2.9 \%$, $\mathrm{p}=0.2$ ). The prevalence of school absence was highest in year 1 and then declined slightly, but was again high in year 6 (Figure 2). Prevalence of absence also varied by weekday, with absence being slightly higher on Mondays and Fridays (Figure 3).

The association between the proportion of children receiving free school meals and absence prevalence is shown in Figure 4. There was no clear correlation between proportion of free school meals and absence $(r=0.5, p=0.29)$.

\section{FIGURE 5}

Illness-related school absence prevalence and laboratory reports of influenza (top panel) and absence incidence and laboratory reports of influenza (bottom panel), United Kingdom, school years 2005-6 and 2006-7
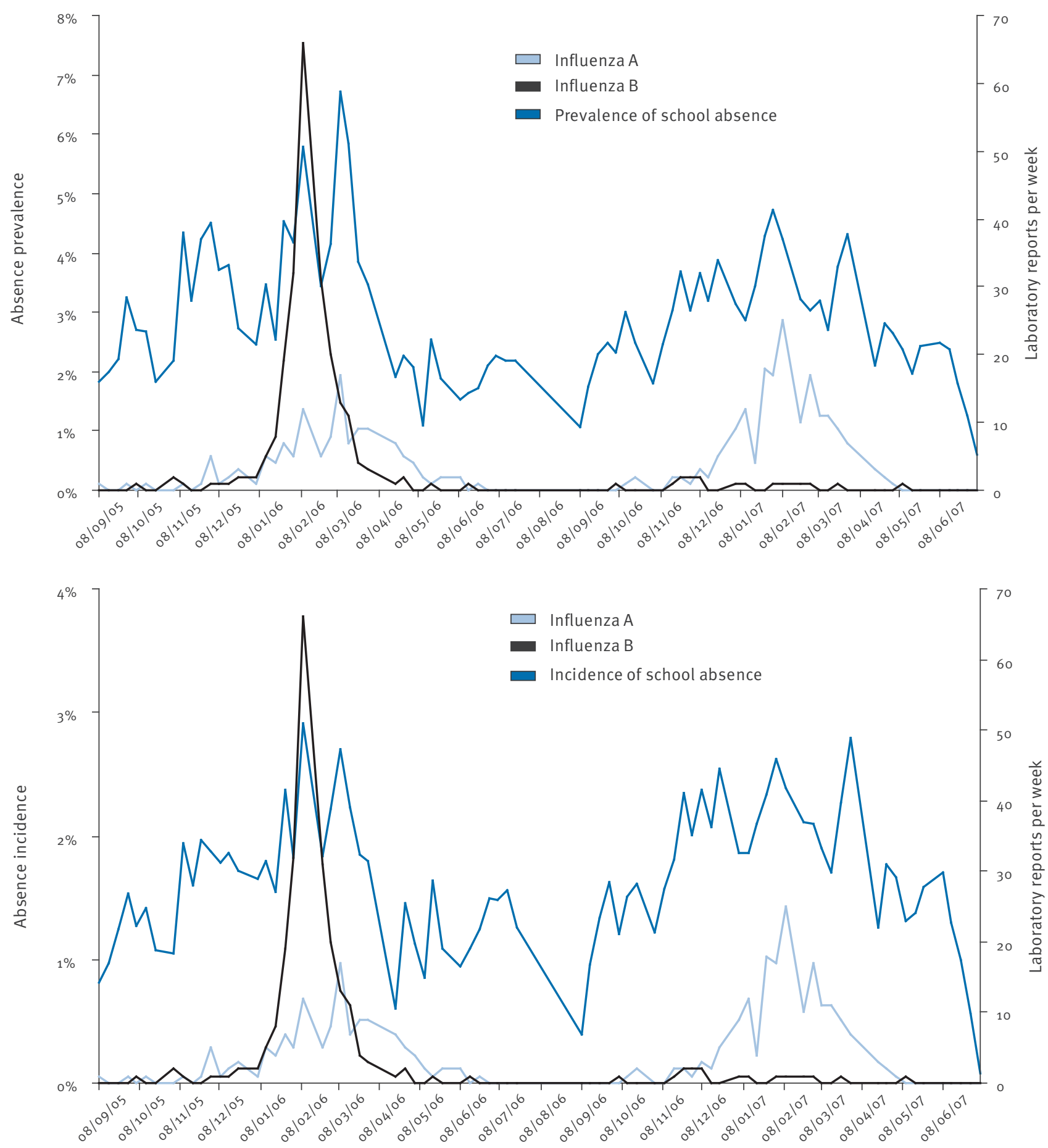
School absence and Influenza activity

The number of laboratory reports of influenza A and B in relation to absence prevalence and incidence over the study period is shown in Figure 5. To smoothen the graphs the number of laboratory reports and the absence data were collapsed at the level of the week. Both influenza seasons covered by the study period were relatively mild. There was a pronounced peak of influenza B in January in the season 2005-6. Influenza $A$ activity was mild in both seasons.
There is some indication that school absence is correlated with influenza laboratory reports. The peak of influenza B in 2005-6 coincided with the peak in absence incidence and was also associated with a peak in absence prevalence. Further, the two peaks of influenza $A$ in the two seasons studied are reflected by peaks in absence incidence. Figure 6 shows the cross-correlation analysis of daily school absence and daily counts of laboratory reports (influenza A and B

\section{FIGURE 6}

Cross-correlation between laboratory reports of influenza and absence prevalence (top panel) and absence incidence (bottom panel), United Kingdom, influenza seasons 2005-6 and 2006-7

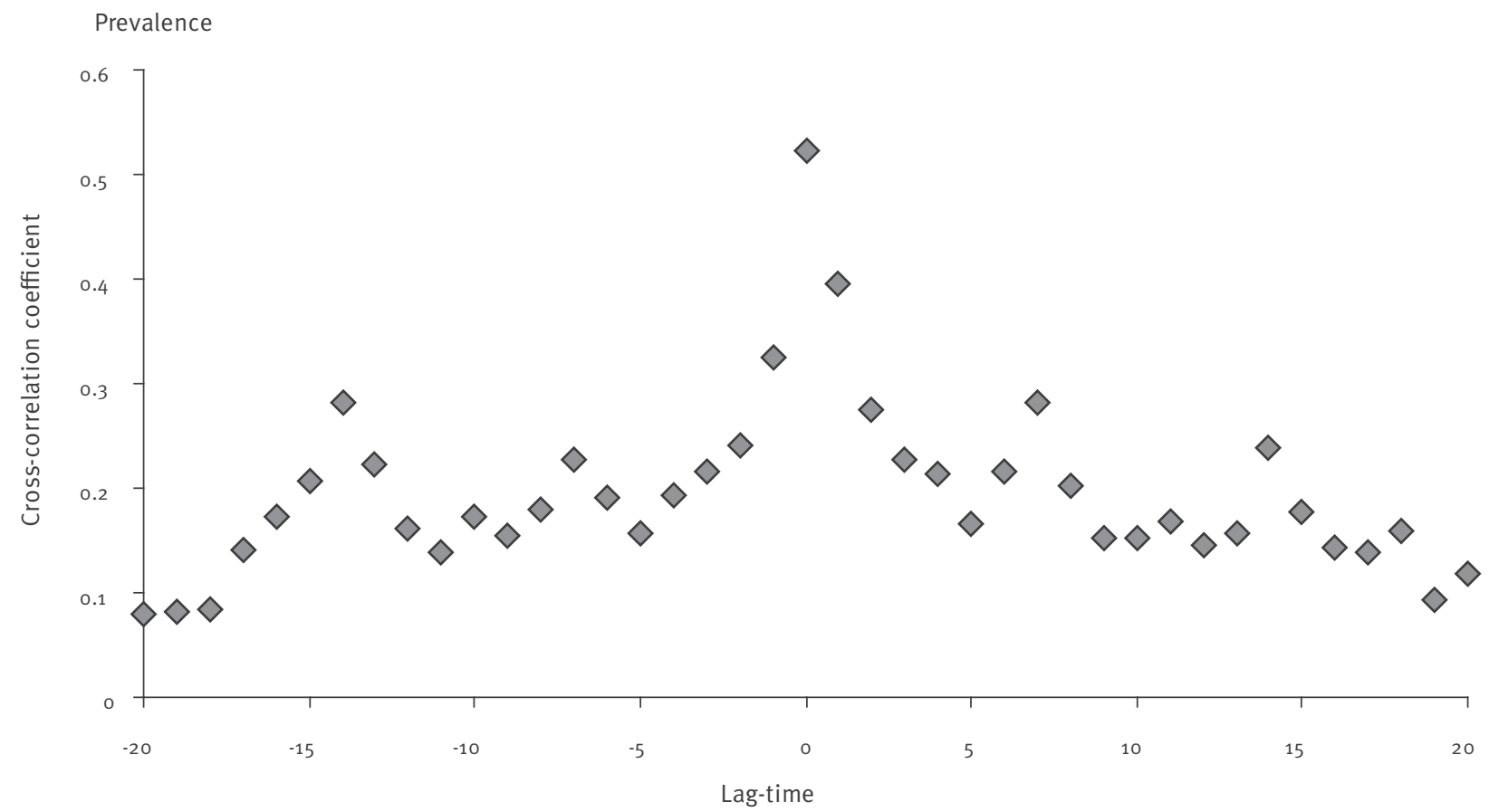

Incidence

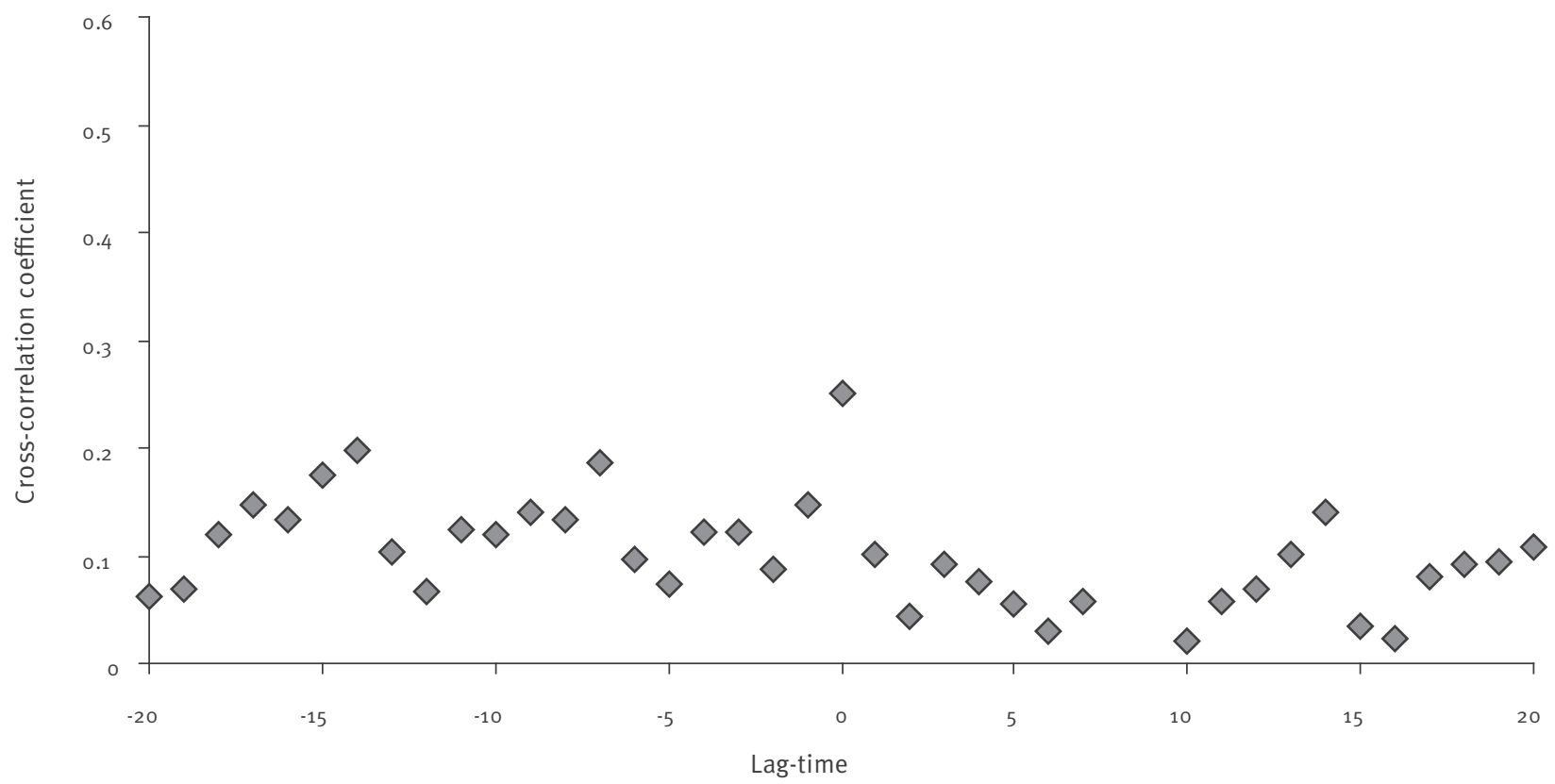


collapsed). For both absence prevalence and incidence there was no apparent lag time in the correlation with influenza laboratory reports. At lag= o the correlation between influenza and prevalence was moderate at 0.52 (p<0.001). For incidence, the coefficient was lower at 0.25 (p<0.001). There was some evidence that during the four weeks of peak Influenza activity, the duration of absence episodes was slightly longer than outside the influenza season (mean absence duration 1.9 days versus 1.8 days outside Influenza activity period, $\mathrm{p}=0.09$ ).
Figure 7 shows the temporal relationship between laboratory reports and school absence, restricted to prevalence data for the year 2006-7, for each of the six individual schools. The peak of influenza A activity is well reflected in the absence prevalence in schools 3 to 6 but not apparent in schools 1 and 2 .

\section{Discussion}

The present analysis provides a basic epidemiological description of school absence for a better understanding of this potential surveillance tool. By analysing data at day-level we were able to calculate figures on school absence prevalence, incidence and episode

\section{FIGURE 7}

Illness-related school absence prevalence for each of the six individual schools in relation to influenza activity, United Kingdom, school year 2006-7
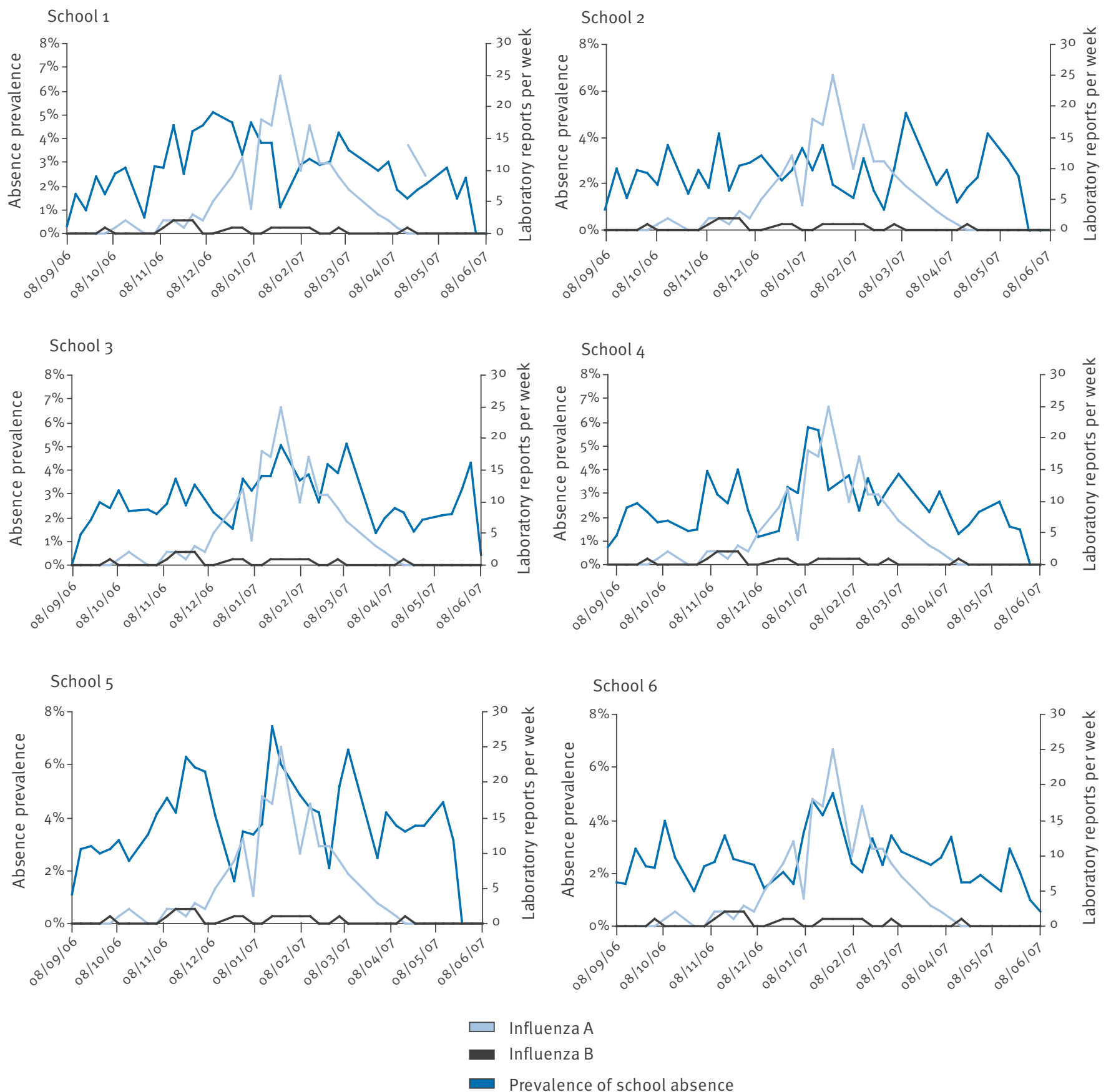
duration. A surprising result was that absence varied relatively little by age, since we had expected the younger years to be absent more frequently due to a presumed higher susceptibility to respiratory infections including influenza $[4,5]$. The duration of absence episodes was slightly longer during the influenza season, and this effect may well have been stronger had influenza activity been greater in the years under study [13]. Absence prevalence may therefore be a more specific proxy for influenza activity because prevalence is affected by absence duration whereas incidence is not. In this analysis we found a much stronger correlation between prevalence and influenza activity than for absence incidence. Respiratory infections caused by a wide range of viruses are very common among schoolaged children. Therefore school absence data may have an equally low specificity as other syndromic surveillance tools such as data from the public helpline of the National Health Service, NHS direct, in the UK which records the number of calls due to respiratory symptoms. As shown in Figure 5, there were several high peaks in absence prevalence and incidence in our data that were not associated with influenza A and B laboratory reports. However, Zaho et al. showed that peaks of absenteeism in individual schools may be due to outbreaks of influenza unnoticed by other surveillance tools [11]. As shown in Figure 7, absenteeism over time can vary considerably between schools even if they are located in the same area. In contrast to Mook et al. we found no evidence that aggregated absence data peak earlier than influenza laboratory reports [12].

Apart from immediacy, school absence records have the advantage that the data are already recorded at school level for legal reasons in the UK. In many countries school attendance is a legal requirement and in those countries school absence data in general are a very complete data source. Absence data provide a denominator of children at risk, which in contrast to many other surveillance tools allows not only to monitor trends, but also to calculate population based prevalence data which can be used to assess the economic and educational impact of influenza. The obvious drawback is that no data are recorded on weekends and during school holidays.

In conclusion, school absence data for influenza surveillance have limitations compared to surveillance tools that provide a continuous data record. Primarily, they may be useful for the early detection of localised school outbreaks and to estimate the socio-economic and educational impact of influenza [12].
3. Vynnycky E, Edmunds WJ. Analyses of the 1957 (Asian) influenza pandemic in the United Kingdom and the impact of school closures. Epidemiol Infect. 2008;136(2):166-79.

4. Fraser C, Donnelly CA, Cauchemez S, Hanage WP, Van Kerkhove MD, Hollingsworth TD, et al. Pandemic potential of a strain of influenza $A\left(\mathrm{H}_{1} \mathrm{~N}_{1}\right)$ : early findings. Science. 2009;324(5934):1557-61.

5. Yang Y, Sugimoto JD, Halloran ME, et al. The transmissibility and control of pandemic influenza $A\left(\mathrm{H}_{1} \mathrm{~N}_{1}\right)$ virus. Science. 2009;326(5953):729-33..

6. Cauchemez S, Valleron AJ, Boelle PY, Flahault A, Ferguson NM. Estimating the impact of school closure on influenza transmission from Sentinel data. Nature. 2008;452(7188):750-4.

7. Cauchemez S, Ferguson NM, Wachtel C, Tegnell A, Saour G, Duncan B, et al. Closure of schools during an influenza pandemic. Lancet Infect Dis. 2009;9(8):473-81.

8. Reichert TA, Sugaya N, Fedson DS, Glezen WP, Simonsen L, Tashiro M. The Japanese experience with vaccinating schoolchildren against influenza. N Engl] Med. 2001;344(12):889-96.

9. Dyer DL, Shinder A, Shinder F. Alcohol-free instant hand sanitizer reduces elementary school illness absenteeism. Fam Med. 2000;32(9):633-8.

10. Rosen L, Manor O, Engelhard D, Brody D, Rosen B, Peleg H, et al. Can a handwashing intervention make a difference? Results from a randomized controlled trial in Jerusalem preschools. Prev Med. 2006;42(1):27-32.

11. Zhao H, Joseph C, Phin N. Outbreaks of influenza and influenza-like illness in schools in England and Wales, 2005/06. Euro Surveill. 2007;12(5):E3-E4. pii=705. Available from: http://www.eurosurveillance.org/ViewArticle. aspx?Articleld $=705$

12. Mook P, Joseph C, Gates P, Phin N. Pilot scheme for monitoring sickness absence in schools during the 2006/07 winter in England: can these data be used as a proxy for influenza activity? Euro Surveill 2007;12(12): E11-E12. pii=755. Available from: http://www.eurosurveillance.org/ViewArticle. aspx?Articleld $=755$

13. Principi N, Esposito S, Gasparini R, Marchisio P, Crovari P; FluFlu Study Group. Burden of influenza in healthy children and their households. Arch Dis Child. 2004;89(11):1002-7.

1. Neuzil KM, Hohlbein C, Zhu Y. Illness among schoolchildren during influenza season: effect on school absenteeism, parental absenteeism from work, and secondary illness in families. Arch Pediatr Adolesc Med. 2002;156(10):986-91.

2. Sullivan KM, Monto AS, Longini IM Jr. Estimates of the US health impact of influenza. Am J Public Health. 1993;83(12):1712-6. 